\title{
Efectos del sedentarismo en niños en edad escolar: revisión sistemática de estudios longitudinales \\ Effects of sedentary school-age children: a systematic review of longitudinal studies

\author{
*José Antonio O rtiz-Sánchez, *Jesús del Pozo-Cruz, *Rosa M aría Alfonso-Rosa, *Daniel Gallardo-Gómez, \\ **Francisco Álvarez-Barbosa \\ *Universidad de Sevilla (España), **CEU Cardenal Spínola (España)
}

\begin{abstract}
Resumen: El objetivo deeste estudio fue conocer quéinvestigaciones de carácter longitudinal se han llevado a cabo que hayan anal izado variables relacionadas con el sobrepeso, la obesidad y el sedentarismo en edad escolar, y qué datos se pueden extraer de las mismas. Se revisaron las siguientes bases de datos: MEDLINE (PubM ed), Scopus, SportDiscus y WOS (Web of Science). Dicha revisión fue realizada siguiendo el modelo PICOS, y a partir de él, se constituyó el protocolo de búsqueda utilizando términos que definan o representen a niños y jóvenes en edad escolar, que puedan presentar o no sobrepeso u obesidad y sedentarismo. Siguiendo el procedimiento Preferred Reporting Items for Systematic Reviews and M eta-Analysis (PRISM A), de los 2237 estudios encontrados tan solo 28 cumplieron todos los criterios, por lo que fueron analizados en su totalidad para poder ser incluidos en la revisión. La calidad metodológica de los estudios se evaluó mediante la escala Newcastle 0 tawa. De los 28 estudios seleccionados finalmente, 13 tenían una calidad metodológica buena, mientras que los 15 restantes tenían una calidad aceptable. Tras realizar la revisión, se puede concluir que el sedentarismo se ve aumentado paulatinamente con la edad mientras que laactividad física decrece, y consecuentemente, esta situación hace que el sobrepeso y la obesidad se vean incrementadas.
\end{abstract}

Palabras clave: niños; escuela; obesidad; sobrepeso; sedentarismo; actividad física.

\begin{abstract}
The objective of this study was to find out what longitudinal researches have been car ried out that have analyzed variables related to overweight, obesity and sedentary lifestyle in school age, and what data can be extracted from them. A systematic review of the MED LINE (PubM ed), Scopus, SportD iscus andW OS (Web of Science) databases was carried out following the PICO s model, using terms that define or represent children and young people of school age, who may or may not be overweight or obese. Following the PRISM A procedure, of the 2237 studies found only 28 met all the criteria and were ther efore analyzed in their entirety for inclusion in the review. The methodological quality of the studies was assessed using the Newcastle-0 tawa scale. Of the 28 studies finally selected, 13 were of good methodological quality, while the remaining 15 were of acceptable qual ity. After review was conducted, it can be concluded that sedentary lifestyle is gradually increased with age while physical activity decreases, and consequently, this situation causes overweight and obesity increase.
\end{abstract}

Keywords: children; school; obesity; overweight; sedentary lifestyle; physical activity.

\section{Introducción}

La O rganización Mundial de la Salud (OMS) define el sobrepeso y la obesidad como la acumulación anormal o excesiva de grasa corporal que puede llegar a ser perjudicial para la salud (OMS, 2016). Se trata de una patología que está caracterizada por un aumento de la masa grasa corporal. Esto es debido al desequilibrio existente entre la ingesta alimenticia y el gasto energético (Moxley et al., 2019). La prevalencia de sobrepeso y obesidad se sitúa en cotas muy altas, y se afirma que en el año 2019 más de 1900 millones de

Fecha recepción: 02-09-20. Fecha de aceptación: 23-11-20

Francisco Álvarez-Barbosa

falvarez@ ceuandalucia.es personas padecían de esta enfermedad, de las cuales más de 650 millones eran obesos (O CDE, 2019). Los datosa nivel europeo no son nada favorables, ya que un estudio realizado en 20 países de la U nión Europea, estima que el $53,1 \%$ de la población padece sobrepeso u obesidad (Marques, Peralta, Naia, Loureiro, \& de Matos, 2018).

Recientes estudios confirman que en Europa aproximadamente el $25 \%$ de la población infantil y juvenil en edad escolar tienen exceso de grasa. Las tasas másaltas de sobrepeso y obesidad infantil se encontraron en los países de la cuenca del mediterráneo; España, Malta, Italia y Grecia (Aranceta-Bartrina, GianzoCitores, \& Pérez-Rodrigo, 2020; O rtiz-Pinto et al., 2020). Esta prevalencia varía según el estatus donde se sitúen las familias, cuánto mayor estatus económico, menores tasas de sobrepeso y obesidad (Sánchez-Cruz, 
de Ruiter, Jiménez-M oleón, García, \& Sánchez, 2018).

El sobrepeso y la obesidad infantil traen consigo una serie de patologías que se asocian a esta demasía de grasa, entre las que podemos encontrar enfermedades como: diabetes, problemas cardiovasculares, problemas locomotores, diferentes tipos de cáncer o problemas de carácter psicológico. Estas enfermedades comúnmente se podían observar en personas adultas y mayores, sin embargo, cada vez se observa con mayor frecuencia en la población infantil y juvenil. Todo esto hace que la esperanza de vida se vea reducida, así como la calidad de la misma (Blüher, 2019; Chooi, Ding, \& Magkos, 2019; Franciscato et al., 2019; H oare, Skouteris, FullerTyszkiewicz, Millar, \& Allender, 2014; Jauregui et al., 2020). La importancia del estudio e investigación del sobrepeso y la obesidad infantil, radica en poder identificar las diferentes comorbilidades con las que pueden estar asociadas. Se ha estimado que en torno al $55 \%$ de la población infantil y el $70 \%$ de los adolescentes con esta patología, la padecerán también en edad adulta, incrementando así el riesgo de sufrir una mayor morbilidad. (Llewellyn, Simmonds, 0 wen, \&Woolacott, 2016). En este mismo sentido encontramos investigaciones que afirman que durante el desarrollo delaprimerainfancia, entre los 5 y los 7 años, comienzan al gunoscambios hormonalesy metabólicos, este periodo es un momento crítico ya que muchos de los menores que inician esta etapa con sobrepeso tienen una mayor probabilidad de que ésta persista hasta la mayoría de edad (M ead, Batterham, Atkinson, \& Ells, 2016).

Estas evidencias negativas para la vida cotidiana de losmenores, también setraduce en un aumento de carga económica para los diferentes gobiernos mundiales, ya que las personas con sobrepeso u obesidad tienen unos costos médicos de un 30\% más que una persona con normopeso (Bleich et al., 2018). En la comunidad Europea, el costo total, tanto directo como indirecto, que se atribuye al sobrepeso y a la obesidad es el equivalente al $0,47-0,6 \%$ del PIB (von Lengerke \& Krauth, 2011). En la actualidad, este exceso de grasa corporal se considera una pandemia y representa uno de los mayores desafíos para los gobiernos y la salud pública (Yáñez-O rtega et al., 2019).

En cuanto al desarrollo del sobrepeso y la obesidad según el género hay opiniones dispares, una reciente publicación de la $O C D E$, confirma que a nivel general existe una mayor preval encia de las mujeres con exceso de peso frente a los hombres (OCDE, 2019), sin embargo, algunos estudios matizan esta afirmación, ya que dependiendo de la zona geográfica a nivel mundial la prevalencia recae sobre un género u otro, siendo en Europa, América y la zona del Pacífico donde existe una mayor prevalencia para el género masculino (Kim \& Shin, 2020).

El aumento desmesurado del sobrepeso y laobesidad, se debe a un conglomerado de factores entre los que podemos encontrar; determinantes genéticos y hereditarios, determinantes de actividad física y sedentarismo, determinantes de hábitos alimenticios, determinantes del sueño, determinantes socioeconómicos y determinantes del entorno, entre otros.

En el desarrollo de esta revisión nos centraremos en los determinantes de actividad física y sedentarismo, que tengan lugar durante un periodo longitudinal.

Desde la segunda mitad del Siglo XX, se han registrado drásticas reducciones en los niveles de actividad física, especialmente en los países industrializados ('erban, 'erban, Butica, \& Lungeanu, 2018). La mayoría de estudios actuales, indican que la realización de actividad física por parte de menores, está en pleno declive (Sallis et al., 2016). A nivel mundial, se estima que sólo una quinta parte de los jóvenes son suficientemente físicamente activos (Straatmann et al., 2019). A esto, se le une el aumento de uso de nuevastecnologías entendidas como televisión, videoconsolas, ordenadores o smarphones, fomentando el desarrollo de un creciente sedentarismo (CzenczekLewandowska et al., 2019; Yang et al., 2019).

En 2007 laO M S puso en marchalalniciativaEuropea deVigilancia de la 0 besidad. Si analizamos los informes dados, podemos observar que la gran mayoría de niños delos distintos países no practican lacantidad de actividad física recomendada y pasan muchas horas sedentarias viendo la televisión o usando aparatos electrónicos (Nittari et al., 2019).

Por tanto, el objetivo de este estudio fue revisar la literatura científica existente sobre la relación que hay entre el mantenimiento del sedentarismo y la actividad física en el tiempo, y el sobrepeso y la obesidad de menores en edad escolar evaluados en estudios longitudinales.

\section{Método}

\section{Fuente de datos y búsqueda}

Para la realización de este estudio, se siguieron las directrices marcadas para las revisiones sistemáticas y meta-análisis PRISM A, la cual incluye una lista con 27 ítems para su verificación y un diagrama de flujo 
compuesto de cuatro fases (M oher, Liberati, Tetzlaff, \& Altman, 2010). Para esta revisión sistemática se realizaron búsquedas en las siguientes bases de datos: MEDLINE (a través de PubM ed), Scopus, SportDiscus, WOS (Web Of Science). La última búsqueda en estas bases de datos se realizó en el mes de abril de 2020.

La búsqueda en las citadas bases de datos fue diseñada respondiendo al acrónimo establecido por el modelo PICO (Landa-Ramírez, 2014), para poder encontrar aquellos estudios longitudinales que investigasen la relación entre el sedentarismo en edad escolar, la actividad física y la prevalencia de sobrepeso y obesidad se utilizó el protocolo de búsqueda que se muestra en el archivo adicional 1 y cuyo número de registro en Prospero esCRD 42020201647. Algunos delostérminos utilizados para definir la población fueron infant, child o youngster. Para definir el sedentarismo se utilizaron términos como sedentary, physical inactivity. Para definir los estudios longitudinales fueron utilizados términos como longitudinal studies o longitudinal survey y, por último, para definir las variables se utilizaron descriptores como physical activity o physical conditioning, overweigth, obesity 0 body mass index.

\section{Selección de estudios}

Los estudios incluidos en la revisión se sel eccionaron siguiendo la metodología PRISMA, utilizada en revisiones sistemáticas y meta-análisis. U navez realizada la búsqueda, se eliminaron los registros duplicados. En el primer cribado se analizaron título y resumen de los registros encontrados, durante la segunda criba se analizaron los textos completos de aquellos estudios potenciales de ser incluidos ya que cumplían todos y cada uno de los criterios de inclusión. Los autores del estudio participaron en todos los pasos descritos de la selección de artículos, en caso de discrepancia se realizaron consultas a expertos externos a la investigación.

Los criterios de inclusión fueron: 1) Edad comprendida entre los 3 y 18 años; 2) participantes integrados en el sistema educativo; 3) sujetos sin patologíasque pudieran afectar alasvariables del estudio; 4) estudios que anal izaran el nivel de actividad física de los mismos sujetos durante periodos superiores a 1 año. Por el contrario, los estudios fueron excluidos si: 1) no eran estudios longitudinales; 2) no se analizaban a los mismossujetos duranteel periodo detiempo establecido; 3) no se estudia el nivel de actividad física de los participantes; 4) no estudian variables relacionadas con el sobrepeso y la obesidad; 5) estudios no publicados en español, inglés o portugués.

Lacalidad metodológicadelosestudios seleccionados fue evaluada mediante la escala N ew castle- 0 ttawa, donde por medio de un sistema de puntuación basado en estrellas, se juzgan los estudios desde tres perspectivas: la selección de los grupos de estudio, la comparabilidad de los grupos y la determinación de la exposición o el resultado de interés para los estudios de casos y controles o los estudios de cohortes, respectivamente (Wells et al., 2013).

\section{Resultados}

\section{Estudios incluidos}

Inicialmente se encontraron 2237 artículos tras la búsqueda en las diferentes bases de datos: MEDLINE ( $n=792)$, Scopus ( $n=899)$, SportDiscus ( $n=159)$ yW OS $(n=387)$. Tras la eliminación de 781 estudios duplicados, 1456 fueron revisados incluyendo su título y resumen. Tras la eliminación de 1400 estudios que no cumplieron alguno de los criterios de inclusión, especialmente aquellos que no seguían las variables establecidas, 56 estudios completos fueron revisados como potenciales estudios para ser incluidos en la revisión sistemática. Finalmente 28 fueron los estudios incluidos en este análisis, ya que 18 artículos no cumplían con el criterio de ser estudios longitudinales, 8 no seguían las variables marcadas, y los 2 restantes no cumplían con el criterio de la edad (Figura 1).

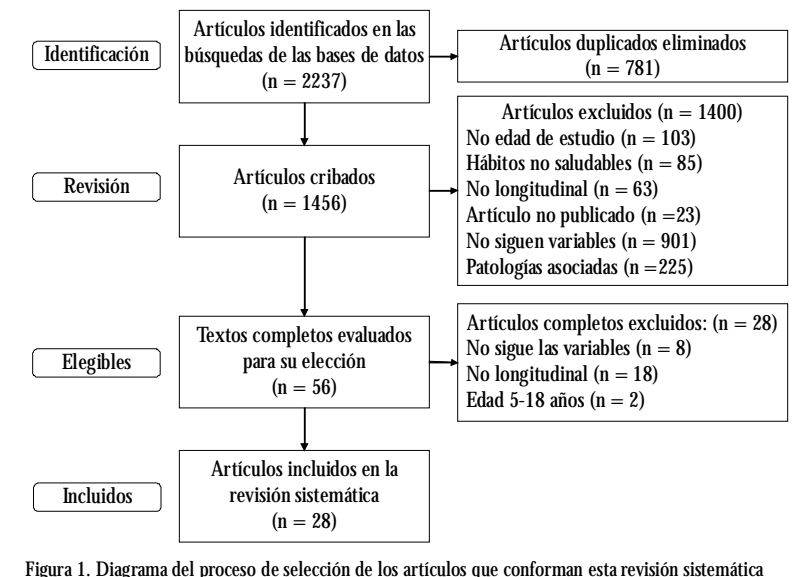

\section{Evaluación de la calidad metodológica}

De acuerdo con la escala N ew castle-O tawa, la metodología de aquellos estudios con una puntuación de 7-10 son considerados «buena calidad», con una puntuación de 5-7 son considerados como «calidad aceptable» y aquellos con una puntación de 1-4 son considerados como «calidad pobre». En la presente 
revisión, 13 artículos tenían una metodología de buena calidad, 16 artículos tenían una calidad aceptable y ninguno tendría una calidad pobre (tabla 1). afirma que la reducción del tiempo sedentario y el aumento de la actividad física, está relacionado con la disminución del sobrepeso.

\begin{tabular}{|c|c|c|c|c|c|c|c|c|}
\hline Estudio & $\begin{array}{c}\text { Representatividad } \\
\text { de la muestra }\end{array}$ & $\begin{array}{l}\text { Tamaño de } \\
\text { la muestra e }\end{array}$ & $\begin{array}{c}\text { No } \\
\text { encuestados }\end{array}$ & $\begin{array}{l}\text { Determinación } \\
\text { s de la exposición }\end{array}$ & Comparación & $\begin{array}{l}\text { Evaluación } \\
\text { del resultado }\end{array}$ & $\begin{array}{c}\text { Prueba } \\
\text { estadística gl }\end{array}$ & $\begin{array}{l}\text { Puntuación } \\
\text { lobal (máx=10) }\end{array}$ \\
\hline 1 Adams et al. (2020) & - & - & - & *** & ** & * & * & 6 \\
\hline 2 Aires et al. (2010) & - & - & - & ** & ** & * & * & 6 \\
\hline 3 Archambault et al. (2018) & - & * & - & ** & ** & * & * & 7 \\
\hline 4 Baquet et al. (2006) & - & - & - & ** & ** & * & * & 6 \\
\hline 5 Basterfield et al. (2015) & - & - & - & $* *$ & ** & * & * & 6 \\
\hline 6 Baxter-Jones et al. (2008) & - & - & - & ** & ** & $*$ & * & 6 \\
\hline 7 Chen et al. (2005) & - & - & - & ** & ** & * & * & 6 \\
\hline 8 Corder et al. (2015) & - & - & - & ** & ** & * & * & 6 \\
\hline 9 Crawford et al. (2010) & - & * & - & ** & ** & * & * & 7 \\
\hline 10 Devís-D evís et al. (2017) & - & - & - & $* *$ & ** & * & * & 6 \\
\hline 11 Elinder et al. (2014) & - & - & - & ** & ** & * & * & 6 \\
\hline 12 Fulton et al. (2009) & - & - & * & ** & ** & * & * & 7 \\
\hline 13H aerens et al. (2010) & - & - & * & ** & ** & * & * & 7 \\
\hline 14 Hamer et al. (2018) & - & * & - & ** & ** & * & * & 7 \\
\hline $15 \mathrm{H}$ arding et al. (2015) & - & - & - & ** & ** & * & * & 6 \\
\hline 16 Jackson et al. (2017) & - & * & - & ** & ** & * & * & 7 \\
\hline 17 Janssen et al. (2016) & - & * & - & $* *$ & ** & * & * & 7 \\
\hline 18 Janz et al. (2017) & - & - & - & ** & ** & * & * & 6 \\
\hline 19 Llargués et al. (2017) & $*$ & - & - & ** & ** & * & * & 7 \\
\hline 20 Mann et a. (2017) & - & - & - & ** & ** & * & * & 6 \\
\hline 21 Marcus et al. (2009) & $*$ & * & - & ** & ** & $*$ & * & 8 \\
\hline 22 Mitchell et al. (2013) & - & - & - & ** & ** & * & * & 6 \\
\hline 23 Murillo et al. (2015) & $*$ & - & - & $* *$ & $* *$ & $*$ & $*$ & 7 \\
\hline 240 morou et al. (2016) & * & - & * & $* *$ & ** & * & * & 8 \\
\hline 250 'Neill et al. (2017) & $*$ & * & - & ** & ** & * & * & 8 \\
\hline 26 Planas et al. (2012) & - & - & - & * & ** & * & * & 5 \\
\hline 27 Saeidlou et al. (2006) & - & * & - & * & ** & * & * & 6 \\
\hline 28Timperio et al (2008) & $*$ & $*$ & - & ** & ** & * & $*$ & 8 \\
\hline
\end{tabular}

Muchos de estos estudios afirman que el crecimiento del tiempo sedentario es debido al uso de nuevas tecnologías, las más frecuentemente utilizadas son la Televisión, Smarphones, ordenadores y videojuegos (tabla 2). Otra de las principales características hallada en esta revisión es que tanto el aumento de tiempo sedentario como la reducción de actividad física hace más incisión en el género femenino. En este mismo sentido, las chicas padecen un porcentaje mayor de grasa corporal y de exceso de peso.

\section{Características de los estudios}

Las características de los 28 artículos incluidos en esta revisión sistemática están resumidas en la tabla 2. En estos estudios participaron muestras de entre $61 \mathrm{y}$ 37750 participantes en los que la participación femenina es mayor que la masculina en 20 de los 28 artículos. Las mediciones más comunes en estos 28 artículos investigados son el sedentarismo, la actividad física y las medidas antropométricas donde se incluye el peso, la talla 0 índice de masa corporal (IMC), entre otras. $\mathrm{La}$ duración de estas investigaciones oscila entre los 2 y los 7 años. La media de duración se sitúa en 2,97 cursos académicos. El 31\% delosestudios relacionan el aumento del sedentarismo y la disminución de la actividad física con un incremento en la prevalencia del sobrepeso y la obesidad, otro $31 \%$ relaciona directamente el aumento del sedentarismo y la disminución de la actividad física con el incremento del IMC, y por lo tanto la variación de los percentiles. Un $14 \%$ relaciona el sedentarismo y la baja actividad física con el aumento de la grasa corporal. Un $11 \%$ de los artículos no encuentra relación entre el sedentarismo, la actividad física y el exceso de peso 0 grasa corporal. El 7\% de los artículos encuentra relación entre el aumento del tiempo sedentario, la disminución de la actividad física y la reducción del sobrepeso y la obesidad. Un $3 \%$ de losartículos concluye que la actividad física no influye en la disminución del sobrepeso y la obesidad. Por último, el 3\% restante
Uno de los resultados que más llama la atención, deriva del tipo de estudios que se han analizado, es decir, estudios longitudinales, estos resultados nos indican que conforme avanza el tiempo y por consiguiente la edad de la muestra, el tiempo sedentario se hace cada vez mayor, mientras que la actividad física se ve mer mada, dando como resultado un aumento del sobrepeso y la obesidad.

\section{Discusión}

El sobrepeso y la obesidad esuna patología compleja, compuesta por múltiples factores y en gran parte prevenibles, que afectaamás de un tercio de lapoblación mundial. Un reciente estudio publicado por la $\mathrm{OCDE}$, muestra que la obesidad infantil sigue creciendo a nivel global, aunque a diferentes ritmos. Este estudio analiza la tendencia marcada en diferentes países desde 1975 a 2018, donde la prevalencia de obesidad y sobrepeso aumentó de manera constante en niños y jóvenes de 5 a 19 años. Esteaumento fue de un promedio de 0,3 puntos porcentuales por año, aunque este crecimiento no fue uniforme, ya que en algunos países esta tendencia fue mayor, mientras que en otros se redujo (OCDE, 2019). Uno de los principales factores que acrecienta este aumento del exceso de peso es la disminución de la actividad física y el incremento del tiempo sedentario (aerban, aerban, Butica, \& Lungeanu, 2020). 
Tabla 2.

Tabla resumen de los estudios que conforman la revision sistentica

\begin{tabular}{|c|c|c|c|c|c|c|}
\hline $\begin{array}{l}\text { Estudios } \\
\text { (Adams \& Jago, } \\
\text { 2020) }\end{array}$ & $\begin{array}{l}\text { Participantes } \\
\text { Primer año: } 1299 \\
\text { Segundo año: } 1223 \\
\text { Tercer año: } 1296\end{array}$ & $\begin{array}{c}\text { Edad } \\
\text { Entre } 5 \text { y } 11 \text { años }\end{array}$ & $\begin{array}{c}\text { Género } \\
\text { Primer año: } \\
\text { Chicos: } 636 \\
\text { Chicas: } 663 \\
\text { Segundo año: } \\
\text { Chicos: } 673 \\
\text { Chicas: } 550 \\
\text { Tercer año: } \\
\text { Chicos: } 674 \\
\text { Chicas: } 662\end{array}$ & $\begin{array}{c}\text { Duración } \\
3 \text { cursos escolares }\end{array}$ & $\begin{array}{l}\text { Parámetros } \\
\text { Actividad Física } \\
\text { Antropometría } \\
\text { Mediciones: } \\
\text { Acelerometría }\end{array}$ & $\begin{array}{c}\text { Resultados } \\
\text { Aumento del sedentarismo y la obesidad con el tiempo. } \\
\text { También aumenta el tiempo de sedentarismo, tanto } \\
\text { durante la semana como el fin de semana. Disminución } \\
\text { del tiempo activo. } \\
\text { Predominan las familias con educación superior. }\end{array}$ \\
\hline Aires et al. (2010) & 345 & Entre 11 y 16 & $\begin{array}{l}\text { Chicos: } 198 \\
\text { Chicas: } 147\end{array}$ & 3 cursos escolares & $\begin{array}{c}\text { Actividad Física } \\
\text { Sedentarismo } \\
\text { Antropometría } \\
\text { Mediciones: Batería FITNESSGRAM Cuestionario }\end{array}$ & $\begin{array}{c}\text { Aumento del sobrepeso, la obesidad y el tiempo } \\
\text { sedentario. Disminuye al AF. }\end{array}$ \\
\hline $\begin{array}{l}\text { Archambault et al. } \\
\qquad(2018)\end{array}$ & 356 & Entre 6 y 9 años & $\begin{array}{l}\text { Chicos: } 182 \\
\text { Chicas: } 174\end{array}$ & 2 cursos escolares & $\begin{array}{c}\text { Sedentarismo } \\
\text { Actividad Física } \\
\text { Antropometría } \\
\text { Mediciones: Acelerometría Báscula de análisis corporal }\end{array}$ & $\begin{array}{l}\text { Incremento del sobrepeso y la obesidad. Incremento del } \\
\text { tiempo sedentario. Decrecimiento del tiempo deAF. }\end{array}$ \\
\hline $\begin{array}{l}\text { Baquet et al. } \\
\quad(2006)\end{array}$ & 193 & Entre 11 y 16 años & $\begin{array}{l}\text { Chicos: } 98 \\
\text { Chicas: } 95\end{array}$ & 4 cursos escolares & $\begin{array}{c}\text { Antropometría } \\
\text { Actividad Física } \\
\text { Mediciones: Báscula de análisis cor poral Test EURO FIT }\end{array}$ & $\begin{array}{c}\text { Incremento del IMC } \\
\text { Sedentarismo y AF se mantienen estables. }\end{array}$ \\
\hline $\begin{array}{l}\text { Basterfield et al. } \\
\qquad(2015)\end{array}$ & $\begin{array}{l}\text { Primer año: } 609 \\
\text { Segundo año: } 585 \\
\text { Tercer año: } 525\end{array}$ & Entre 6 y 13 años & $\begin{array}{l}\text { Primer año: } \\
\text { Chicos:307 } \\
\text { Chicas:302 } \\
\text { Segundo año: } \\
\text { Chicos:293 } \\
\text { Chicas:292 } \\
\text { Tercer año: } \\
\text { Chicos:268 } \\
\text { Chicas:257 }\end{array}$ & 3 cursos escolares & $\begin{array}{c}\text { Antropometría } \\
\text { Actividad Física } \\
\text { Sedentarismo } \\
\text { Participación en clubes deportivos } \\
\text { Mediciones: Acelerometría Báscula de análisis corporal }\end{array}$ & $\begin{array}{l}\text { Aumento del IMC, de la grasa corporal y del tiempo de } \\
\text { sedentarismo. Aumento de la participación en clubes } \\
\text { deportivos. Disminución de laAF. }\end{array}$ \\
\hline $\begin{array}{l}\text { Baxter-Jones et al. } \\
\text { (2008) }\end{array}$ & 222 & Entre 8 y 15 años & $\begin{array}{l}\text { Chicos:113 } \\
\text { Chicas:109 }\end{array}$ & 6 cursos escolares & $\begin{array}{c}\text { Antropometría } \\
\text { Actividad Física } \\
\text { Sedentarismo } \\
\text { Mediciones: Absorción de rayos } X \text { de doble energía } \\
\text { Cuestionarios }\end{array}$ & $\begin{array}{l}\text { La masa de grasa corporal aumenta con la edad. LaAF } \\
\text { tiene una influencia significativa cuando se alcanza la } \\
\text { madurez biológica. }\end{array}$ \\
\hline Chen et al. (2005) & 7794 & Entre 9 y 12 años & $\begin{array}{l}\text { Chicos: } 3925 \\
\text { Chicas: } 3869\end{array}$ & 3 cursos escolares & $\begin{array}{c}\text { Calidad de vida } \\
\text { Actividad Física } \\
\text { Sedentarismo } \\
\text { Mediciones: Cuestionarios }\end{array}$ & $\begin{array}{c}\text { Aumento del sedentarismo y disminución de laAF. Los } \\
\text { estilos de vida desfavorables en la infancia muestran una } \\
\text { baja calidad de vida. }\end{array}$ \\
\hline $\begin{array}{l}\text { Corder et al. } \\
(2015)\end{array}$ & $\begin{array}{l}\text { Primer año: } 2064 \\
\text { Segundo año: } 1019 \\
\text { Tercer año: } 480\end{array}$ & Entre 9 y 14 años & $\begin{array}{l}\text { Primer año: } \\
\text { Chicos: } 1148 \\
\text { Chicas:916 } \\
\text { Segundo año: } \\
\text { Chicos: } 519 \\
\text { Chicas: } 500 \\
\text { Tercer año: } \\
\text { Chicos: } 257 \\
\text { Chicas:223 }\end{array}$ & 3 cursos escolares & $\begin{array}{c}\text { Actividad Fsísica } \\
\text { Sedentarismo } \\
\text { Antropometría } \\
\text { Mediciones: Acelerometría Báscula de anáisis corporal }\end{array}$ & $\begin{array}{c}\text { Disminución del IMC, del número de personas obesas y } \\
\text { con sobrepeso y de la grasa corporal. } \\
\text { Aumento del tiempo de sedentarismo y disminución del } \\
\text { tiempo de AF. }\end{array}$ \\
\hline $\begin{array}{l}\text { Crawford et al. } \\
\text { (2010) }\end{array}$ & 301 & Entre 10 y 17 años & $\begin{array}{l}\text { Chicos: } 173 \\
\text { Chicas: } 128\end{array}$ & 3 cursos escolares & $\begin{array}{c}\text { Actividad Física } \\
\text { Sedentarismo } \\
\text { Antropometría } \\
\text { A mbiente del hogar } \\
\text { Mediciones: Acelerometría Cuestionarios Báscula de } \\
\text { análisis corporal }\end{array}$ & $\begin{array}{l}\text { El IMC aumenta en ambos sexos, aunque en los chicos hay } \\
\text { una ligera disminución entre la línea base y el primer } \\
\text { seguimiento. Disminución de laAF y aumento del } \\
\text { sedentarismo. El papel de la familia es más importante } \\
\text { que el del vecindario. }\end{array}$ \\
\hline $\begin{array}{l}\text { (Devís-D evís et al., } \\
\text { 2017) }\end{array}$ & 755 & Entre 11 y 16 años & $\begin{array}{l}\text { Chicos: } 407 \\
\text { Chicas: } 348\end{array}$ & 2 cursos escolares & $\begin{array}{c}\text { Actividad Física } \\
\text { Sedentarismo } \\
\text { Antropometría } \\
\text { Mediciones: Cuestionarios Báscula de análisis corporal }\end{array}$ & $\begin{array}{c}\text { El porcentaje de obesidad y sobrepeso está disminuyendo. } \\
\text { No se ha demostrado que laAF influya en esto. }\end{array}$ \\
\hline $\begin{array}{c}\text { (Elinder, } \\
\text { Heinemans, } \\
\text { Zeebari, \& } \\
\text { Patterson, 2014) }\end{array}$ & 813 & Entre 8 y 15 años & $\begin{array}{l}\text { Chicos: } 406 \\
\text { Chicas: } 407\end{array}$ & 2 cursos escolares & $\begin{array}{c}\text { Actividad Física } \\
\text { Antropometría } \\
\text { Salud } \\
\text { Mediciones: Cuestionarios }\end{array}$ & $\begin{array}{c}\text { En general, la obesidad y el sobrepeso disminuyen, y la } \\
\text { obesidad infantil aumenta parcial mente. } \\
\text { LaAF disminuye. }\end{array}$ \\
\hline $\begin{array}{l}\text { (Fulton et al., } \\
\text { 2009) }\end{array}$ & $\begin{array}{c}472 \\
\text { Primer año: } 212 \\
\text { Segundo año: } 151 \\
\text { Tercer año:109 }\end{array}$ & Entre 13 y 18 años & $\begin{array}{l}\text { Primer año: } \\
\text { Chicos: } 114 \\
\text { Chicas: } 98 \\
\text { Segundo año: } \\
\text { Chicos: } 77 \\
\text { Chicas: } 74 \\
\text { Tercer año: } \\
\text { Chicos: } 54 \\
\text { Chicas: } 55\end{array}$ & 3 cursos escolares & $\begin{array}{c}\text { Antropometría } \\
\text { Actividad Física } \\
\text { Sedentarismo } \\
\text { Mediciones: Cuestionarios Báscula de análisis corporal }\end{array}$ & $\begin{array}{l}\text { LaAF aumenta en los niños, disminuye en las niñas. } \\
\text { Aumento parcial del sedentarismo en ambos. El IMC } \\
\text { aumenta en los chicos, parcialmente en las chicas. }\end{array}$ \\
\hline $\begin{array}{l}\text { Haerens et al. } \\
\text { (2010) }\end{array}$ & $\begin{array}{l}\text { Primer año: } 1270 \\
\text { Segundo año: } 1066 \\
\text { Tercer año: } 791 \\
\text { Cuarto año: } 585 \\
\end{array}$ & Entre 10 y 14 años & $\begin{array}{l}\text { Chicos: } 611 \\
\text { Chicas: } 659\end{array}$ & 4 cursos escolares & $\begin{array}{c}\text { Actividad Física } \\
\text { Sedentarismo } \\
\text { Antropometría } \\
\text { Mediciones: Cuestionarios }\end{array}$ & $\begin{array}{c}\text { La disminución del consumo de frutas, de laAF, de las } \\
\text { horas de educación física y el aumento del sedentarismo } \\
\text { lleva a un incremento del IMC. }\end{array}$ \\
\hline $\begin{array}{l}\text { Hamer et al. } \\
\text { (2018) }\end{array}$ & 4770 & $\begin{array}{c}\text { Entre } 7 \text { y } 14 \\
\text { personas }\end{array}$ & $\begin{array}{l}\text { Chicos: } 2329 \\
\text { Chicas: } 2441\end{array}$ & 2 cursos escolares & $\begin{array}{c}\text { Actividad Física } \\
\text { Antropometría } \\
\text { Mediciones: Acelerometría, Báscula de análisis cor poral } \\
\text { Cuestionario }\end{array}$ & $\begin{array}{l}\text { Aumento del IMC. Cuanto más AF vigorosa menos } \\
\text { probabilidad de padecer sobrepeso u obesidad. }\end{array}$ \\
\hline $\begin{array}{l}\text { Harding et al. } \\
\quad(2015)\end{array}$ & 363 & Entre 12 y 15 años & $\begin{array}{l}\text { Chicos: } 223 \\
\text { Chicas: } 140\end{array}$ & 2 cursos escolares & $\begin{array}{c}\text { Sedentarismo } \\
\text { Actividad Física } \\
\text { Antropometría } \\
\text { Mediciones: Acelerometría, Báscula de análisis cor poral } \\
\text { Estadiómetro }\end{array}$ & $\begin{array}{l}\text { El IMC aumenta con el tiempo, al igual que la obesidad, } \\
\text { mientras que el sobrepeso disminuye. El tiempo de } \\
\text { sedentarismo aumentay laAF disminuye. }\end{array}$ \\
\hline $\begin{array}{l}\text { Jackson et al. } \\
\text { (2017) }\end{array}$ & 4938 & Entre 5 y 14 años & $\begin{array}{l}\text { Chicos: } 2528 \\
\text { Chicas: } 2410\end{array}$ & 4 cursos escolares & $\begin{array}{c}\text { Actividad Física } \\
\text { Sedentarismo } \\
\text { Estilos de vida } \\
\text { Antropometría } \\
\text { Mediciones Cuestionarios }\end{array}$ & $\begin{array}{l}\text { Aumento del IMC. El tiempo de visualización de pantalla } \\
\text { está asociado con el aumento de peso. Disminución de la } \\
\text { AF. Las conductas relacionadas con la salud no tienen } \\
\text { efecto sobre el peso. }\end{array}$ \\
\hline
\end{tabular}


Tabla resumen de los estudios que conforman la revisión sistemática

\begin{tabular}{|c|c|c|c|c|c|c|}
\hline Estudios & Participantes & Edad & Género & Duración & Parámetros & Resultados \\
\hline $\begin{array}{l}\text { Janssen et al. } \\
\text { (2016) }\end{array}$ & $\begin{array}{l}\text { Primer año: } 507 \\
\text { Segundo año: } 510 \\
\text { Tercer año: } 425 \\
\text { Cuarto año: } 310\end{array}$ & Entre 7 y 15 años & $\begin{array}{l}\text { Primer año: } \\
\text { Chicos 252 } \\
\text { Chicas 255 } \\
\text { Segundo año: } \\
\text { Chicos 265 } \\
\text { Chicas 245 } \\
\text { Tercer año: } \\
\text { Chicos 227 } \\
\text { Chicas } 198 \\
\text { Cuarto año: } \\
\text { Chicos 166 } \\
\text { Chicas 144 }\end{array}$ & 4 cursos escolares & $\begin{array}{c}\text { Sedentarismo } \\
\text { M ediciones: Acelerometría }\end{array}$ & $\begin{array}{l}\text { Aumento del sedentarismo en cada etapa del estudio. } \\
\text { Disminución de laAF. }\end{array}$ \\
\hline Janz et al. (2017) & 463 & Entre 5 y 19 años & $\begin{array}{l}\text { Chicos } 233 \\
\text { Chicas } 230\end{array}$ & 7 cursos escolares & $\begin{array}{l}\text { Antropometría y evaluación de la madurez. } \\
\text { Actividad Física } \\
\text { Adiposidad } \\
\text { Ver latelevisión } \\
\text { M ediciones: A celerometría Báscula de análisis corporal } \\
\text { Estadiómetro Absorción de rayos } X \text { de doble energía }\end{array}$ & $\begin{array}{l}\text { Aumento de la grasa corporal y del IMC. Tiempo de } \\
\text { televisión estable. La tendencia general de laAF es } \\
\text { disminuir. Aumento parcial del sedentarismo. }\end{array}$ \\
\hline $\begin{array}{l}\text { Llargués et al. } \\
\text { (2017) }\end{array}$ & $\begin{array}{l}\text { Primer año: } 566 \\
\text { Segundo año: } 509 \\
\text { Tercer año: } 426 \\
\text { Cuarto año: } 397\end{array}$ & Entre 7 y 12 años & $\begin{array}{l}\text { Chicos } 234 \\
\text { Chicas } 332\end{array}$ & 4 cursos escolares & $\begin{array}{c}\text { Actividad Física } \\
\text { Antropometría } \\
\text { Mediciones: Báscula de análisis cor poral Estadiómetro } \\
\text { Cuestionarios }\end{array}$ & $\begin{array}{l}\text { El grupo de control hace menosAF y tiene más tiempo de } \\
\text { sedentarismo. El exceso de peso corporal aumenta. }\end{array}$ \\
\hline Mann et a. (2017) & $\begin{array}{l}\text { Primer año: } 502 \\
\text { Segundo año: } 506 \\
\text { Tercer año: } 420 \\
\text { Cuarto año: } 306\end{array}$ & Entre 6 y 16 años & $\begin{array}{l}\text { Primer año: } \\
\text { Chicos 241 } \\
\text { Chicas 261 } \\
\text { Segundo año: } \\
\text { Chicos 262 } \\
\text { Chicas 244 } \\
\text { Tercer año: } \\
\text { Chicos 223 } \\
\text { Chicas } 197 \\
\text { Cuarto año: } \\
\text { Chicos 163 } \\
\text { Chicas 143 }\end{array}$ & 4 cursos escolares & $\begin{array}{c}\text { Actividad Física } \\
\text { Antropometría } \\
\text { Sedentarismo } \\
\text { M ediciones: Acelerometría Báscula de análisis corporal } \\
\text { Estadiómetro }\end{array}$ & $\begin{array}{l}\text { Aumento del IMC, de la grasa corporal y del tiempo de } \\
\text { sedentarismo. Disminución de laAF. }\end{array}$ \\
\hline $\begin{array}{l}\text { Marcus et al. } \\
\text { (2009) }\end{array}$ & $\begin{array}{l}\text { Primer año: } 1390 \\
\text { Segundo año: } 1021\end{array}$ & Entre 6 y 10 años & $\begin{array}{l}\text { Primer año: } \\
\text { Chicos } 684 \\
\text { Chicas } 706 \\
\text { Segundo año: } \\
\text { Chicos } 504 \\
\text { Chicas } 517\end{array}$ & 2 cursos escolares & $\begin{array}{c}\text { Actividad Física } \\
\text { Antropometría } \\
\text { Mediciones: Acelerometría Báscula de análisis cor poral } \\
\text { Cuestionario }\end{array}$ & $\begin{array}{l}\text { Aumento del sobrepeso y la obesidad en el grupo de } \\
\text { control, disminución en el grupo de intervención LaAf es } \\
\text { mayor en el grupo de intervención. }\end{array}$ \\
\hline $\begin{array}{l}\text { Mitchell et al. } \\
\text { (2013) }\end{array}$ & $\begin{array}{l}\text { Primer año: } 740 \\
\text { Segundo año: } 681 \\
\text { Tercer año: } 540 \\
\text { Cuarto año: } 424\end{array}$ & Entre 9 y 15 años & & 4 cursos escolares & $\begin{array}{c}\text { Actividad Física } \\
\text { Antropometría } \\
\text { Sedentarismo } \\
\text { Mediciones: Acelerometría, Estadiómetro Láser para el } \\
\text { peso }\end{array}$ & $\begin{array}{l}\text { Aumento del tiempo de sedentarismo y disminución de la } \\
\text { AF. Asociación entre el aumento del tiempo de } \\
\text { sedentarismo y el aumento del IMC. }\end{array}$ \\
\hline $\begin{array}{l}\text { Murillo et al. } \\
\text { (2015) }\end{array}$ & $\begin{array}{c}682 \\
\text { Primer año: } 229 \\
\text { Segundo año: } 227 \\
\text { Tercer año: } 226\end{array}$ & Entre 12 y 15 años & $\begin{array}{c}\text { Primer año: } \\
\text { Chicos } 108 \\
\text { Chicas } 121 \\
\text { Segundo año: } \\
\text { Chicos } 92 \\
\text { Chicas 135 } \\
\text { Tercer año: } \\
\text { Chicos } 113 \\
\text { Chicas } 113\end{array}$ & 3 cursos escolares & $\begin{array}{c}\text { Actividad Física } \\
\text { Sedentarismo } \\
\text { Mediciones: Acelerometría Cuestionarios }\end{array}$ & $\begin{array}{l}\text { En general, el sedentarismo está en alza. Hay diferencias } \\
\text { significativas entre diferentes grupos. }\end{array}$ \\
\hline $\begin{array}{l}\text { O morou et al. } \\
\text { (2016) }\end{array}$ & 1445 & & $\begin{array}{l}\text { Chicos } 823 \\
\text { Chicas } 622\end{array}$ & 2 cursos escolares & $\begin{array}{c}\text { Actividad Física } \\
\text { Antropometría } \\
\text { Sedentarismo } \\
\text { Calidad de vida } \\
\text { Mediciones: Cuestionarios }\end{array}$ & $\begin{array}{c}\text { Existe una asociación entre la calidad de vida y laAF. Una } \\
\text { baja calidad de vida está relacionada con un alto nivel de } \\
\text { sedentarismo. }\end{array}$ \\
\hline $\begin{array}{l}\text { O'Neill et al. } \\
\text { (2017) }\end{array}$ & $\begin{array}{l}\text { Primer año: } 8570 \\
\text { Segundo año: } 7423\end{array}$ & Entre 9 y 13 años & & 2 cursos escolares & $\begin{array}{c}\text { Sedentarismo } \\
\text { Actividad Física } \\
\text { Antropometría } \\
\text { Mediciones: Cuestionarios Báscula de análisis corporal }\end{array}$ & $\begin{array}{l}\text { Por lo general, aumenta el sobrepeso, pero disminuye la } \\
\text { obesidad. Los niños participan más que las niñas en laAF. } \\
\text { Altos niveles de sedentarismo. }\end{array}$ \\
\hline Planas et al. (2012) & 61 & Entre 12 y 18 años & $\begin{array}{l}\text { Chicos } 38 \\
\text { Chicas } 23\end{array}$ & 2 cursos escolares & $\begin{array}{c}\text { Actividad Física } \\
\text { Antropometría } \\
\text { Mediciones: Báscula de análisis cor poral }\end{array}$ & $\begin{array}{l}\text { Reducción del sobrepeso y mejora de laAF. } \\
\text { Empeoramiento de los hábitos alimenticios. }\end{array}$ \\
\hline $\begin{array}{l}\text { Saeidlou et al. } \\
\text { (2006) }\end{array}$ & 37.750 & Entre 12 y 15 años & $\begin{array}{l}\text { Chicos } 17.386 \\
\text { Chicas } 20.364\end{array}$ & 2 cursos escolares & $\begin{array}{c}\text { Antropometría } \\
\text { Actividad Física } \\
\text { Sedentarismo } \\
\text { Mediciones: Báscula de análisis cor poral Cuestionarios }\end{array}$ & $\begin{array}{l}\text { Aumento del sobrepeso, la obesidad y el tiempo } \\
\text { sedentario. Disminución de la actividad física. }\end{array}$ \\
\hline $\begin{array}{l}\text { Timperio et al } \\
\text { (2008) }\end{array}$ & 344 & Entre 10 y 15 años & $\begin{array}{l}\text { Chicos } 192 \\
\text { Chicas } 152\end{array}$ & 2 cursos escolares & $\begin{array}{c}\text { Sedentarismo } \\
\text { Actividad Física } \\
\text { Antropometría } \\
\text { Mediciones: Báscula de anál isis corporal Cuestionarios }\end{array}$ & $\begin{array}{l}\text { El sedentarismo está asociado con el aumento del IMC. } \\
\qquad \text { AF disminuye. }\end{array}$ \\
\hline $\begin{array}{l}\text { Toriola et al. } \\
\text { (2015) }\end{array}$ & 283 & $\begin{array}{c}\text { Entre } 14 \text { y } 16 \\
\text { años }\end{array}$ & $\begin{array}{l}\text { Chicos } 172 \\
\text { Chicas } 111\end{array}$ & 2 cur sos escolares & $\begin{array}{c}\text { AF } \\
\text { Antropometría } \\
\text { M ediciones: } \\
\text { Cinta métrica para cintural cadera } \\
\text { Test EURO FIT Cuestionario }\end{array}$ & $\begin{array}{l}\text { Aumento del sobrepeso y la obesidad. Aumento de la } \\
\text { grasa corporal. }\end{array}$ \\
\hline
\end{tabular}

Los resultados obtenidos en esta revisión sistemática nos muestran que el tiempo sedentario aumenta paulatinamente con los años. Este aumento de tiempo sedentario hace que tanto el sobrepeso como la obesidad se vean incrementados, afectando a ambos sexos, 21 de los 28 artículos analizados afirman y demuestran un incremento tanto en el IMC, la grasa corporal como en el sobrepeso y la obesidad. El desarrollo de la prevalencia de sobrepeso y obesidad durante los 2-3 primeros años de vida, tiene más riesgo de persistir en estamisma condición durante los 4-5 años, y decontinuar en la misma dinámica en los 6-7 años, además la 
probabilidad de mantener el exceso de peso aumenta de 6 a 16 veces más (M ead et al., 2016). La prevalencia de sobrepeso y obesidad se eleva más rápido durante la transición de la etapa infantil hacia la etapa juvenil y principio de la adultez que durante cualquier otro periodo de edad (Corder $\&$ W inpenny, 2020).

Es sabido que la infancia y la adolescencia es un período tanto de crecimiento físico como intelectual, sin embargo, también es un periodo que se caracteriza por la asunción de comportamientos poco saludables como la inactividad física o el sedentarismo. (Dubuc, Aubertin-Leheudre, \& Karelis, 2020). Además, estetipo de comportamientostienen un fuerte elemento habitual, por lo tanto, es más probable que se mantengan a lo largo del tiempo (Straatmann et al., 2019). En relación con esta afirmación, y analizando la tabla 2, se puede observar como la mayoría de los artículos incluidos en esta revisión afirman un decrecimiento de la actividad física, mientras que el tiempo sedentario se ve aumentado. Según Jago et al. el nivel de práctica de actividad física va cambiando según la edad, aumentando entre los 3 y los 6 años, donde al canza su punto máximo de participación, coincidiendo con la edad de escolarización primaria. A partir de este momento, el tiempo dedicado a realizar actividad física comienza a disminuir y, por ende, empieza a aumentar el tiempo sedentario de forma lineal entre las edades de 6 y 15 años. Esta disminución de actividad física y aumento de sedentarismo se ve más acrecentada para el género femenino que para el masculino (Jago et al., 2020; Schwarzfischer et al., 2018). Esta última afirmación coincide con los resultados obtenidos en esta revisión, donde la mayoría de artículos afirman que las chicas realizan menos actividad física y tienen un mayor índice de sedentarismo que los chicos. U no de los principales motivos con los que se justifica la menor práctica de actividad física por parte del género femenino viene dada por los estereotipos y la falsa premisa de la masculinización a través del deporte (Bisquert Bover, BallesterArnal, Gil Llario, Elipe M iravet, \& López Fando Galdón, 2020; 0 viedo et al., 2013).

En este creciente sedentarismo, la mayor parte del tiempo inactivo en los más jóvenes está dedicado al tiempo que pasan delante de una pantalla, entendido este en la amplitud de dispositivos existentes actualmente como televisión, Smartphone, ordenadores u otros dispositivos inteligentes (Bassett, John, Conger, Fitzhugh, \& Coe, 2015). Un reciente estudio longitudinal realizado en varias provincias de España ha demostrado que el tiempo de pantallaesel factor que más ha afectado al desarrollo de la obesidad en la muestra analizada (Bawaked et al., 2020). Por otra parte, el estudio AVENA, muestra datos muy parecidos a los expuestos anteriormente, donde el factor más importante para el aumento de peso es el tiempo que la muestra pasaba delante de una pantall a seguido de la baja actividad física, la poca frecuencia de comidas y la omisión del desayuno (Schröder et al., 2017). Un meta-análisis realizado en 2016 dejó en evidenciaque cada hora adicional de tiempo de pantalla supone aumentar el riesgo de obesidad infantil en un 13\% (Zhang,W u, Zhou, Lu, \& Mao, 2016). Estas líneas de investigación que unen el tiempo sedentario y el tiempo de pantalla, coinciden con la mayoría de artículosincluidos en esta revisión, los cuales exponen queel aumento de tiempo sedentario es debido en gran parteal entretenimiento ofrecido por los nuevos dispositivos electrónicos.

Para combatir este aumento desmesurado de peso y las consecuencias de su padecimiento, se antoja decisivo la adherencia a comportamientos saludables como actividad física, reducir el tiempo que pasan delante de unapantalla, buenaalimentación, rutinas de sueño (Berlin et al., 2017; Kovács et al., 2015). Son conocidos los múltiples beneficios que aporta la práctica de ejercicio físico parael bienestar y la salud de las personas (Purslow, Hill, Saxton, Corder, \& Wardle, 2008; Remmers et al., 2017), por lo que se deben establecer desde los estamentos políticos, educativos y sociales una serie de medidas donde se promuevan y ofrezcan actividades físicas variadas que hagan disminuir el tiempo sedentario.

A pesar de los datos expuestos, es necesario tener en cuenta al gunas limitaciones a la hora de realizar una interpretación de los resultados, dado que no existen muchos estudios de carácter longitudinal que aborden los efectos de la disminución de la actividad física y el creciente sedentarismo en la obesidad y el sobrepeso.

\section{Conclusión}

A raíz de los resultados proyectados por los estudios que conforman la presente revisión, se puede concluir que el tiempo dedicado a la realización de actividad física disminuye conforme se avanza cronológicamente, mientras que las actividades sedentarias se incrementan en este mismo periodo de tiempo. A su vez, se hace visible un aumento de la prevalencia del sobrepeso y la obesidad. Por lo tanto, se puede deducir que cuanto más cerca de la adultez están los alumnos en edad escolar más riesgos corren de dedicar su tiempo a actividades sedentarias y por ende el aumento de la grasa corporal. 
El uso de las nuevas tecnologías se antoja como uno de los principales promotores de este aumento del tiempo sedentario. Estos, junto aotros, son losprincipales motivos del aumento de la prevalencia de sobrepeso y obesidad a nivel mundial.

Es necesario la realización y el análisis de más estudios de carácter longitudinal, para así tener la posibilidad de conocer la evolución de estas variables, poder compararla con estudios anteriores y proponer soluciones adecuadas a esta problemática.

\section{Referencias}

Adams, J., \& Jago, R. (2020). Association of BM I category with change in children's physical activity between ages 6 and 11 years: alongitudinal study. Int J Environ ResPublic H ealth, 44(1), 104-113. doi:10.3390/ ijerph1623465210.1038/ s41366-0190459-0

Aranceta-Bartrina, J., Gianzo-Citores, M., \& Pérez-Rodrigo, C. (2020). Prevalence of overweight, obesity and abdominal obesity in the Spanish population aged 3 to 24 years. TheEN PE study. Rev Esp Cardiol (Engl Ed), 73(4), 290-299. doi:10.1016/ j.rec.2019.07.023

Bassett, D. R. , J ohn, D., Conger, S. A., Fitzhugh, E. C., \& Coe, D. P. (2015). Trends in Physical Activity and Sedentary Behaviors of U nited StatesYouth. J Phys Act H ealth, 12 (8), 1102-1111. Retrieved from http:// search.ebscohost.com/

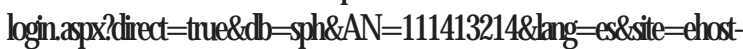
live\&scope =site

Bawaked, R.A., Fernández-Barrés, S., N avar rete-Muñoz, E. M., González-Palacios, S., Guxens, M., Irizar, A., . . . Romaguera, D. (2020). Impact of lifestyle behaviors in early childhood on obesity and cardiometabolic risk in children: Resultsfrom the Spanish INM A birth cohort study. Pediatr Obes, 15(3). doi:10.1111/ ijpo. 12590

Berlin, K. S., Kamody, R. C., Thurston, I. B., Banks, G. G., Rybak, T. M., \& Ferry, R. J. (2017). Physical Activity, Sedentary Behaviors, and Nutritional Risk Profilesand Relationsto Body $M$ ass Index, $O$ besity, and $O$ verweight in Eighth Grade. Behavioral Medicine, 43(1), 31-39. doi:10.1080/ 08964289.2015.1039956

Bisquert Bover, M., Ballester Arnal, R., Gil Llario, M. D., Elipe Miravet, M. , \& López Fando Galdón, M. (2020). Motivaciones parael ejercicio físico y su relación con la salud mental y física: un análisis desde el género. Revista IN FAD de Psicología. International Journal of Developmental and Educational Psychology., 1(1), 351-360. doi:10.17060/ ijodæep.2020.n1.v1.1792

Bleich, S. N., Vercammen, K.A., Zđz, L.Y., Frelier, J. M ., Ebbeling, C. B. \& Peeters, A. (2018). Interventions to prevent global childhood overweight and obesity: a systematic review. Lancet Diabetes Endocrinol, 6(4), 332-346. doi:10.1016/ s22138587(17)30358-3

Blüher, M. (2019). 0 besity: global epidemiology and pathogenesis. $N$ at RevEndocrinol, 15(5), 288-298. doi:10.1038/ s41574-0190176-8

Chooi,Y. C. , Ding, C., \& Magkos, F. (2019). Theepidemiology of obesity. Metabolism, 92, 6-10. doi:10.1016/ j.metabol.2018.09.005

Corder, K., \& W inpenny, E. M. (2020). Becoming a parent: A systematic review and meta-analysis of changes in BMI, diet, and physical activity. 21(4), el2959. doi:10.1111/ obr.12959

Czenczek-Lewandowska, E., Leszczak, J., Weres, A., Baran, J., Wyszyñska, J., Grzegorczyk, J., . . M azur,A. (2019). Sedentary behaviors in children and adolescents with type 1 diabetes, depending on the insulin therapy used. Medicine (Baltimore), 98(19), e15625. doi:10.1097/ md.0000000000015625

Devís-Devís, J., Lizandra, J., Valencia Peris, A., Pérez-Gimeno, E., García-M assò, X., \& Peiró-Velert, C. (2017). Longitudinal changes in physical activity, sedentary behavior and body mass index in adolescence: M igrations towards different weight cluster. PLoS O ne, 12 (6). doi:10.1371/ journal. pone. 0179502

Dubuc, M. M ., Aubertin-Leheudre, M ., \& Karelis, A. D. (2020). Lifestyle HabitsPredictA cademic Performancein High School Students: The Adolescent Student Academic Performance Longitudinal Study (ASAP). Int I Environ Res Public H ealth, 17(1). doi:10.3390/ ijerph17010243

Elinder, L. S., Heinemans, N. , Zeebari, Z., \& Patterson, E. (2014). Longitudinal changes in health behaviours and body weight among Swedish school children - associations with age, gender and parental education - the SCIP school cohort. BM C Public Health, 14. doi:10.1186/ 1471-2458-14-640

Franciscato, S. J., Janson, G., M achado, R., Lauris, J. R. P., de Andrade, S. M. J. , \& Fisberg, M. (2019). Impact of thenutrition education Program N utriamigos $®$ on levels of awarenesson healthy eating habitsin school-aged children. Journal of $\mathrm{H}$ uman Growth and Development, 29(3), 390-402. doi:10.7322/ jhgd. v29.9538

Fulton, J. E., Dai, S., Steffen, L. M., Grunbaum, J. A., Shah, S. M., \& Labarthe, D. R. (2009). Physical Activity, Energy Intake, Sedentary Behavior, andAdiposity inYouth. AmJ Prev M ed, 37 (1 SUPPL.), S40-S49. doi:10.1016/ j.amepre.2009.04.010

Hoare, E., Skouteris, H., Fuller-Tyszkiewicz, M., Millar, L., \& Allender, S. (2014). Associations between obesogenic risk factorsand depression among adolescents: A systematic review. Obesity Reviews, 15 (1), 40-51. doi:10.1111/ obr.12069

J ?uregui, A., Salvo, D., Garc?a-O Ivera, A., Villa, U., T ?llez-Rojo, M. M., Schnaas, L. M., ... Cantoral, A. (2020). Physical activity, sedentary time and cardiometabolic health indicators among Mexican children. Clin Obes 10(1). doi:10.1111/ cob. 12346

Jago, R., Salway, R., Emm-Collison, L., Sebire, S. J., Thompson, J. L., \& Lawlor, D. A. (2020). Association of BM I category with change in children's physical activity between ages 6 and 11 years: a longitudinal study. Int J Obes, 44(1), 104-113. doi:10.1038/ s41366-019-0459-0

Kim, K.-B., \& Shin, Y.-A. (2020). Males with 0 besity and O verweight. Journal of 0 besity \& M etabolic Syndrome, 29(1), 1825. doi:10.7570/ jomes20008

Kovács, E., Hunsberger, M., Reisch, L., Gwozdz,W., Eiben, G., De Bourdeaudhuij, I., . . . M olnár, D. (2015). Adherence to combined lifestyle factors and their contribution to obesity in theIDEFICSstudy. ObesRev, 16 Supp 2, 138-150. doi:10.1111/ obr.12349

Landa-Ramírez, E. D. J., A. (2014). Herramienta PICO parala 
formulación y búsqueda de preguntas clínicamenterelevantes en lapsicooncologíabasadaen laevidencia. PSICOON COLOGÍA. , 11(2-3), 259-270. doi:DO I: 10.5209

Llewellyn, A., Simmonds, M., O wen, C. G., \&Woolacott, N. (2016). Childhood obesity as a predictor of morbidity in adulthood: a systematic review and meta-analysis. O bes Rev, 17 (1), 56-67. doi:10.1111/obr.12316

Marques, A., Peralta, M ., N aia, A., Loureiro, N. , \& de Matos, M. G. (2018). Prevalence of adult overweight and obesity in 20 European countries, 2014. EurJ Public Health, 28(2), 295-300. doi:10.1093/ eurpub/ ckx143

Mead, E., Batterham, A. M., Atkinson, G., \& Ells, L. J. (2016). Predicting future weight status from measurements made in early childhood: a novel longitudinal approach applied to Millennium Cohort Study data. Nutr Diabetes, 6(3), e200. doi:10.1038/ nutd. 2016.3

Moher, D., Liberati, A., Tetzlaff, J., \& Altman, D. G. (2010). Preferred reporting items for systematic reviews and metaanalyses: the PRISMA statement. Int J Surg, 8(5), 336-341. doi:10.1016/ j.jju.2010.02.007

M oxley, E., Habtzghi, D., Klinkhamer, N., Wang, H., Donnelly, S., \& Dykhuizen, J. (2019). Prevention andTreatment of Pediatric $O$ besity: A Strategy Involving Children, Adolescentsand the Family for Improved Body Composition. J Pediatr N urs, 45, 13-19. doi:10.1016/ j.pedn.2018.12.010

Nittari, G., Scuri, S., Petrelli, F., Pirillo, I., di Luca, N. M., \& Grappasonni, I. (2019). Fighting obesity in children from European World Health O rganization member states. Epidemiological data, medical-social aspects, and prevention programs. Clin Ter, 170(3), e223-e230. doi:10.7417/ ct. 2019.2137

OCDE. (2019). The heavy burden of obesity.

http:/ / www. oecd. org/ health/ the heawy-burden-of-obesity-67450d67en. htm.

O MS. (2016). O rganización M undial de la Salud. http:/ / www. who. int/ mediacentrel factsheets/ fs311/ es/. Retrieved from http:/ / www. who. int/ mediacentre/ factsheets/ fs311/ es/ .

O rtiz-Pinto, M. A., O rtiz-Marrón, H., Rodríguez-Rodríguez,A., Casado-Sánchez, L., Cuadrado-Gamarra, J. I. \& G Galán, I. (2020). Parental perception of child health statusand quality of life associated with overweight and obesity in early childhood. Qual LifeRes, 29(1), 163-170. doi:10.1007/ s11136-019-023137

0 viedo, G., Sánchez, J., Castro, R., Calvo, M., Sevilla, J. C., I glesias, A., \& Guerra, M. (2013). Niveles de actividad físicaen población adolescente: estudio de caso. Retos: nuevastendencias en educación física, deportey recreación(23), 43-47.

Purslow, L. R., Hill, C., Saxton, J., Corder, K., \&Wardle, J. (2008). Differencesin physical activity and sedentary time in relation to weight in 8-9 year old children. International Journal of Behavioral N utrition and Physical Activity, 5. doi:10.1186/ 14795868-5-67

Remmers,T.,Thijs, C., Timperio,A., Salmon, J., Veitch, J., Kremers, S. P. J., \& Ridgers, N. D. (2017). DailyW eather and Children's Physical Activity Patterns. M ed Sci SportsExerc, 49(5), 922-929. doi:10.1249/ mss.0000000000001181

Sallis, J. F., Bull, F., Guthold, R., Heath, G.W., Inoue, S., Kelly, P.,
... Hallal, P. C. (2016). Progressin physical activity over the O lympic quadrennium. Lancet, 388(10051), 1325-1336. doi:10.1016/ s0140-6736(16)30581-5

Sánchez-Cruz, J. J., de Ruiter, I., J iménez-M oleón, J. J., García, L. \& \& Sánchez, M. J. (2018). Stabilization and reversal of child obesity in Andalusia using objective anthropometric measures by socioeconomic status. BMC Pediatr, 18(1), 322. doi:10.1186/ s12887-018-1295-4

Schröder, H., Bawaked, R.A., Ribas-Barba, L., Izquierdo-Pulido, M., Roman-Viñas, B., Fíto, M ., \& Serra-Majem, L. (2017). CumulativeEffect of $O$ besogenic Behaviourson Adiposity in Spanish Children and Adolescents. ObesFacts, 10 (6), 584-596. doi:10.1159/000480403

Schwarzfischer, P., Gruszfeld, D., Socha, P., Luque, V., ClosaM onasterolo, R., Rousseaux, D., ... Grote, V. (2018). Longitudinal analysis of physical activity, sedentary behaviour and anthropometric measures from ages 6 to 11 years. International Journal of Behavioral N utrition and Physical Activity, 15(1). doi:10.1186/ s12966-018-0756-3

aerban, C. L., aerban, D. M., Butica, S. I., \& Lungeanu, D. (2020). Web of causation between dietary patterns and childhood obesity: Applying hill's criteria. Romanian Journal of Diabetes, Nutrition and Metabolic Diseases, 25(4), 431-438. doi:10.2478/ rjdnmd-2018-0052

'erban, C. L., 'erban, D. M., Butica, ' I . \& \& Lungeanu, D. (2018). Web of Caustion between Dietary Patterns and Childhood $O$ besity:Applying Hill's Criteria. Romanian Journal of D iabetes Nutrition and Metabolic Diseases, 25(4), 431-438. doi:10.2478/ rjdnmd-2018-0052

Straatmann, V. S. , Almquist,Y. B., O liveira, A. J.,Veiga, G.V., Rostila, M., \& Lopes, C. S. (2019). Stability and bidirectional relationship between physical activity and sedentary behaviours in Brazilian adolescents: Longitudinal findings from aschool cohort study. PLoS One, 14(1), e0211470. doi:10.1123/ jpah.2017-058710.1371/ journal. pone. 0211470

von Lengerke, T., \& Krauth, C. (2011). Economic costs of adult obesity: a review of recent European studies with a focus on subgroup-specific costs. Maturitas, 69(3), 220-229. doi:10.1016/ j.maturitas.2011.04.005

Wells, G., Shea, B., O 'Connell, D. , J, P., Welch, V., Losos, M., \& Tugwell, P. (Producer). (2013). The N ewcastle-O ttawaScale (NOS) for assessing the quality of nonrandomised studies in meta-analyses. Retrieved from http:/ / www.ohri.cal programs/ clinical_epidemiology/ oxford. asp

Yang, L., Cao, C. , Kantor, E. D., N guyen, L. H., Zheng, X., Park, Y.,... Cao,Y. (2019).Trendsin Sedentary BehaviorAmong the US Population, 2001-2016. Jama, 321(16), 1587-1597. doi:10.1001/ jama 2019.3636

Yáñez-O rtega, J. L., ArrietaCerdán, E., Lozano-Alonso, J. E., Gil Costa, M., Gutiérrez-Araus, A. M., Cordero-Guevara, J. A., \&VegaAlonso,T. (2019). Prevalence of overweight and obesity in child population. A study of a cohort in Castile and Leon, Spain. Endocrinol DiabetesN utr, 66(3), 173-180. doi:10.1016/ j.endinu.2018.10.004

Zhang, G., W u, L. , Zhou, L., Lu, W., \& Mao, C. (2016). Television watching and risk of childhood obesity: ameta analysis. Eur J PublicH ealth, 26(1), 13-18. doi:10.1093/ eurpub/ ckv213 